\title{
Begebenheiten unitarischer Geschichte: Von Puritanern zu engagierten Bildungsbürgern
}

\author{
Dan McKanan
}

Unitarismus wird oft über abstrakte und universelle Grundsätze definiert, wie z.B. „Freiheit, Vernunft und Toleranz“ oder auch als „,Wert und Würde, die jeder Person innewohnen“. Doch wie jede Tradition wurde auch der Unitarismus geprägt durch seine kulturelle Umgebung sowie durch zufällige Begebenheiten und Umstände. Diese geschichtlichen Gegebenheiten bilden den Körper des Geistes liberaler Religion. Ohne sie hätten wir möglicherweise keine Institutionen oder Gemeinden, um unsere Grundsätze an kommende Generationen weiterzugeben.

Dieser Beitrag untersucht fünf accidents der Geschichte, die den Unitarismus in den Vereinigten Staaten geprägt haben, Ereignisse und Begebenheiten, die sich als Zufälle betrachten lassen und doch nachhaltige Konsequenzen nach sich zogen: seinen Ursprung aus den puritanischen Kirchen Neuenglands; seine Geburt gleich nach der amerikanischen Revolution, als die junge Nation ihr Experiment der Religionsfreiheit wagte; die prägende Begegnung mit dem deutschen Idealismus zur Zeit Emersons; seine symbiotische Beziehung zu Hochschulen und Universitäten und seine Verbindung mit der Tradition der Universalisten im Jahr 1961. Diese Schilderungen sollen die deutschen Unitarier dazu anregen, diese Begebenheiten zu reflektieren, die ihre eigene Geschichte prägen - sowie auch ihre Zukunft.

Es wäre ebenso möglich wie verführerisch, religiösen Liberalismus über abstrakte Grundsätze zu definieren, ohne einen Bezug zu bestimmten geographischen oder geschichtlichen Zusammenhängen herzustellen. Unitarische Universalisten von heute berufen sich auf sieben Grundsätze; sie reichen von „Wert und Würde, die jeder Person innewohnen“ bis zum „Respekt vor der ineinandergreifenden Vernetzung jeglicher Existenz, zu der wir gehören“. Bill Sinkford, ehemaliger Präsident der Unitarian Universalist Association, hat bekanntlich festgestellt, dass die 
unitarischen Grundsätze nicht ein einziges Wort enthalten, das traditionell als religiös eingestuft werden kann. Doch er hätte noch weiter gehen sollen, bis hin zu der Feststellung, dass diese Grundsätze überhaupt nicht ausdrücklich in irgendeiner Religion oder kulturellen Tradition verwurzelt sind.

Religiöse Liberale im Allgemeinen und Unitarier im Besonderen neigen zu abstrakten Auslegungen und Erläuterungen unserer Traditionen, weil wir befürchten, dass eine Vergangenheit, der man zu viel Aufmerksamkeit schenkt, eine zu große Macht über die Gegenwart gewinnen könnte. Ralph Waldo Emerson beklagte, dass „historisches“ Christentum „,von der Offenbarung als etwas [redet], das schon lange vorbei ist - als ob Gott tot sei“". Wahre Religion, so wie Emerson sie verstand, gründete in der „,intuitive[n] Erkenntnis der Tugend“, die er als zugleich „göttlich“ und „vergöttlichend“ bezeichnete. ${ }^{1}$ Emersons Freund Theodore Parker mahnte die Unitarier, sich von „Formen und Lehren" zu verabschieden, und sich zu konzentrieren auf „dauernde“ Grundsätze wie „das göttliche Leben der Seele, [...] die Liebe zu Gott und den Menschen“. „Wenn [...] bewiesen werden könnte“, so Parker, „daß Jesus von Nazareth nie gelebt hätte, so würde das Christenthum dennoch fest stehen und nichts Übles zu fürchten haben". ${ }^{2}$ Damit wir uns nicht missverstehen: Beide, Emerson wie Parker, wurden von den meisten auch ihrer unitarischen Zeitgenossen als Ketzer angesehen. Aber ihre Worte überdauerten, ebenso wie die wirkungsmächtigen Grundformen unitarischen Glaubens, die auch heutzutage in Amerika fortleben. Auch wir fürchten eine tyrannische Herrschaft der Vergangenheit; auch wir glauben, dass gute Grundsätze alles sind, was wir benötigen, um einen lebendigen Glauben aufrechtzuerhalten.

Trotzdem möchte ich darauf hinweisen, dass abstrakte Grundsätze allein nicht genug sind, um eine lebendige religiöse Bewegung zu erhalten. Der Geist verlangt nach Fleisch, selbst wenn er immer über dessen Grenzen hinausdrängen wird. Das Ewige verlangt nach dem Kurzlebigen. Emersons und Parkers etwas konservativerer Freund Frederic Henry Hedge, selbst das wichtigste Bindeglied zwischen amerikanischem Transzendentalismus und deutschem Idealismus, erläuterte seinen Standpunkt in einer wortgewandten Predigt zur Metapher des heiligen Paulus vom Buchstaben, der töte, und dem Geist, der lebendig mache (2. Kor 3,6):

Der Geist sucht im Verhältnis zu der ihm innewohnenden Kraft, sich zu verkörperlichen, und strebt im Laufe der Zeit danach, zum Buchstaben zu erstarren. [...] Alle Offenbarungen und Entwicklungen erreichen ein Stadium der Verfestigung, nachdem sie einen flüssigen oder gasförmigen $\mathrm{Zu}$ stand durchlaufen haben: Nach dem zeitweisen Leben und Arbeiten als

\footnotetext{
${ }^{1}$ Um die Vortragsform beizubehalten, wird hier zumeist auf einzelne Belege und Nachweise von Zitaten verzichtet. Zu Emersons Divinity School Address vgl. die deutsche Übersetzung von Heiko Fischer (mit einer Einleitung von Dieter Schulz): Drei Ansprachen. Über Bildung, Religion und Henry David Thoreau. Freiburg/Br. 2007, S. 21-49, hier S. 61, 64, 55 u. 57.

2 Theodore Parker: Das Vergängliche und das Bleibende im Christentbume. In: T.P.: Sämmtliche Werke. Erster Band. Kritische und vermischte Schriften. Übersetzt von Johannes Ziethen. Leipzig 1854, S. 167-208, hier S. 173 u. 189
} 
körperloser Geist kristallisieren sie sich zu festen, formalisierten Kräften oder Stützen, stabilisieren sich als Schriften oder Kirchen. [...] Es gibt keinen Widerstreit mit dem Buchstaben: der Geist verlangt ihn.

Hedge hat nicht versucht, dem Vergangenen wieder Geltung zu verschaffen, sondern er hat nur einfach seinen Freund dazu ermahnt, es nicht ganz zu vernachlässigen. Wenn wir unsere liberalen Grundsätze wertschätzten, so Hedge, sollten wir der Art und Weise Aufmerksamkeit schenken, in der diese Grundsätze in den einzelnen Gemeinden mit ihren besonderen Geschichten umgesetzt werden.

Und so möchte ich heute mit Ihnen die besondere Geschichte der unitarischen Tradition teilen, die mir am geläufigsten ist: des Unitarismus in Amerika. Meine Erzählung dieser Geschichte betont nicht die abstrakten Grundsätze, sondern einige Umstände und Faktoren, die es diesen Grundsätzen ermöglichten, körperliche Gestalt zu gewinnen. Damit möchte ich Sie einladen, auch die Ereignisse zu reflektieren, in denen der deutsche Unitarismus das getan hat.

\section{Puritanismus in Neuengland}

Die unitarische Tradition Amerikas entstand in den ersten Dekaden des 19. Jahrhunderts; sie baute auf Fundamenten auf, die zwei Jahrhunderte früher von den puritanischen Gründern von Neuengland gelegt worden waren. Selbst heute noch erinnern sich viele Gemeinden Unitarischer Universalisten mit Stolz an ihre puritanischen Wurzeln; so auch die Gemeinde, der ich angehöre.

Die Verbindung zwischen Puritanismus und Unitarismus mag überraschend sein, weil die Puritaner ganz sicher keine religiösen Liberalen waren. Sie sahen sich dem reformierten Glauben von Johannes Calvin und Ulrich Zwingli verpflichtet und glaubten, dass die Anglikanische Kirche einer „Reinigung“ von katholischen Praktiken und Riten bedürfe. Ihre Glaubenslehre betonte die vollständige Verderbtheit der menschlichen Natur, und sie waren (als wollten sie ebendiese Verderbtheit demonstrieren) gewaltsam intolerant gegenüber jeglicher religiösen Abweichung. Amerikanische Ureinwohner, Quäker, Menschen, die wegen Hexerei angeklagt waren: sie wurden von den Gründern Neuenglands umgebracht. Personen, die auch nur die kleinsten Abweichungen vom Puritanismus predigten, wurden verbannt, unter ihnen Roger Williams, der erste amerikanische Verfechter der Religionsfreiheit. Unitarier können und wollen auf diese Aspekte des puritanischen Erbes nicht stolz sein; vielmehr müssen sie stetig daran arbeiten, diese von den Vorgängern verursachten Wunden zu heilen.

Ein anderer Aspekt des puritanischen Erbes aber ist in unitarischen Gemeinden noch immer lebendig, und das ist die Unabhängigkeit des Gemeindelebens, die protestantische Selbstverwaltung. Die puritanische Cambridge Platform (ein Text, der über viele Jahrzehnte hinweg eine Art Verfassung des kongregationalistischen Gemeinwesens in Neuengland bildete) erklärte 1649, dass jede lokale Gruppe von 
Christen eine Kirche gründen könne, sofern sie nur eine bindende Verpflichtung einginge, im Dienste der Allgemeinheit und gegenseitiger Unterstützung zu handeln. Eine solche Kirche sollte nicht der Autorität eines Bischofs oder einer klerikalen Instanz unterstellt sein, sondern frei ihre eigenen Regeln aufstellen und ihre eigenen Pfarrer benennen.

Es war eine Konsequenz dieses Bekenntnisses zu autonomen Gemeinden, dass sich die puritanischen Kirchen Neuenglands auf den Weg zu einem einzigartigen Experiment in der Geschichte des Christentums machten. Wie viele Kirchen auch in Europa waren sie ,bewährt ' und etabliert: erhielten Steuereinnahmen und kooperierten mit staatlichen Institutionen. Soziologisch ausgedrückt waren sie „Kirchen“ und keine „Sekten“. Sie sahen sich in der Verantwortung nicht nur ihren eigenen Mitgliedern, sondern der gesamten Gesellschaft gegenüber, und so entwickelten sie starke Institutionen, einschließlich Harvard, der ersten und einflussreichsten Universität in Amerika. Dies ebnete den Weg auch für den Aufstieg des Unitarismus; denn es war in Harvard, wo die Puritaner die liberalen Ideen der Aufklärung aufzunehmen begannen. Zur selben Zeit waren die puritanischen Kirchen dem Staat eher auf lokaler als auf nationaler Ebene verbunden und unterstanden auch nicht der Autorität von Bischöfen. Das machte es leichter für sie, sich gegen die Autorität von Königen zu wehren. In der puritanischen Tradition zeichnet sich eine Kirche im Gegensatz zu einer Sekte durch die ausdrückliche Bereitschaft aus, sich für politische oder ökonomische Institutionen zu engagieren und sie notfalls auch zu kritisieren.

Noch heute sind Unitarische Universalisten stolz auf ihre Tradition eines unabhängigen Gemeindewesens; nach wie vor befassen sich unsere Studenten mit den Worten der Cambridge Platform. Obwohl wir nicht länger Steuerzuwendungen erhalten, ist uns das Wohl der Gesellschaft insgesamt ein wichtiges Anliegen. Viele von uns sind aktiv in der Friedensbewegung, kämpfen für wirtschaftliche Gerechtigkeit, für die Umwelt, für die Rechte von Immigranten und die Freiheit von Personen mit unterschiedlichsten sexuellen Identitäten. In unseren Aktivitäten zeigt sich, dass wir gewissenhafte Erben der Puritaner sind.

\section{Amerikanische Revolution und religiöse Freiheit}

Das zweite Ereignis in der unitarischen Geschichte Amerikas ist die Geburt des unitarischen Glaubensbekenntnisses nur wenige Jahre nach der amerikanischen Revolution. Frühe Unitarier, und tatsächlich alle Erben der Puritaner, gehörten zu den enthusiastischsten Unterstützern der Revolution, zum Teil weil sie der Anglikanischen Kirche verübelten, wie sie sich in Neuengland neue Geltung zu verschaffen versuchte, zum Teil auch weil sie skeptisch waren gegenüber jeder Art von zentraler Autorität. Entsprechend war die erste Kirche in Neuengland, die sich selbst „unitarisch“ nannte, die Kings' Chapel, die bis zur Revolution anglikanisch gewesen war. Viele ihrer Mitglieder widersetzten sich der Revolution und wander- 
ten nach Kanada oder England aus. Die Dagebliebenen engagierten einen talentierten jungen Puritaner, James Freeman, der sie überzeugte, folgende beiden Elemente aus ihren Ritualen zu streichen: die Gebete für den König und die Bezüge auf die Dreifaltigkeit. Kurz darauf änderten einige der bekanntesten Pfarrer puritanischer Kirchen ihre Meinung und entwickelten eigene unitarische Vorstellungen; das Ergebnis war die Geburt einer neuen Glaubensgemeinschaft.

Ironischerweise unterstützten die Unitarier die revolutionären Prinzipien der religiösen Freiheit nicht von Anfang an. Diesem Prinzip wurde im ersten Zusatz zur Verfassung der Vereinigten Staaten Ausdruck verliehen, dem First Amendment, das besagt, dass der „Kongress keine Gesetze beschließen darf, die religiöse Institutionen betreffen oder ihre freiheitliche Existenz verbieten". Zunächst bezog sich dies nur auf die Regierung der Föderation: Staaten konnten ihre eigenen etablierten Religionen beibehalten; der unitarisch dominierte Staat Massachusetts tat das auch länger als alle anderen Staaten. Die Unitarier wollten ihre Privilegien nicht aufgeben - aber sie wollten auch ihre Verantwortung für das Wohl der Gesellschaft nicht abgeben. Nachdem jedoch die Veränderung der Machtverhältnisse im Jahr 1830 auch Massachusetts erreicht hatte, erkannten die meisten Unitarier deren Wert und fanden neue Wege, ihrer sozialen Verantwortung gerecht zu werden.

Diese Ereignisse prägen bis heute die Praxis der Unitarischen Universalisten. Für uns ist „Demokratie“ nicht nur ein politischer oder patriotischer Wert, sondern sie ist ein zentraler Punkt unserer Religion selbst. Wir sind stolz auf die demokratischen Entscheidungsformen, die sowohl die regionalen Versammlungen als auch die Hauptversammlung der gesamten Glaubensgemeinschaft kennzeichnen. Zwar sind weniger als ein Promille der amerikanischen Gesamtbevölkerung Unitarische Universalisten, aber immerhin vier von vierundvierzig US-Präsidenten waren Unitarier. Unitarische Universalisten leisten heute in vielen politischen Institutionen ihren Beitrag, vornehmlich in Bereichen, die sich mit dem demokratischen Prozess beschäftigen. In den Staaten Kalifornien und Minnesota beispielsweise arbeiten unitarisch-universalistische Staatssekretäre - in einem Amt also, das verantwortlich ist für die Durchführung von Wahlen; beide haben sich bundesweit verdient gemacht in der Entwicklung von Strategien zur Erhöhung der Wahlbeteiligung und zur Sicherstellung, dass auch tatsächlich jede Stimme gezählt wird. Andere Unitarische Universalisten unterstützen die Redefreiheit und eine möglichst breite Mitwirkung an demokratischen Prozessen etwa durch Organisationen wie die American Civil Liberties Union und die League of Women Voters, eine aus der frühen Frauenbewegung hervorgegangene Vereinigung, die sich seitdem für die Rechte aller Wähler einsetzt. Beide Organisationen wurden von Unitariern im frühen 20. Jahrhundert gegründet. 


\section{Die Transzendentalisten entdecken den Deutschen Idealismus}

Die erste große Kontroverse in der Geschichte der amerikanischen Unitarier vollzog sich in den 1830er und 1840er Jahren, als eine neue Generation von Pfarrern eine neue Glaubenslehre einführte, die von der Philosophie des deutschen Idealismus inspiriert war. Diese Geschichte begann mit Frederic Henry Hedge, dem Sohn eines Harvard-Professors für Germanistik, der große Teile seiner Kindheit in Deutschland verbracht und ausgezeichnete Kenntnisse in dieser Sprache und Kultur erworben hatte. Ich habe ihn eben schon einmal zitiert: Er war es, der darauf bestand, dass der „Geist" sich immer wieder in „Buchstaben“ verkörperlichen müsse. Als junger Mann teilte Hedge die Auffassungen, die er aus dem deutschen Idealismus und der deutschen Romantik gelernt hatte, mit seiner Kindheitsfreundin Margaret Fuller, der mit Abstand gebildetsten Frau des damaligen Amerika. Gemeinsam überzeugten sie R. W. Emerson, Theodore Parker und viele andere, dass man sich ernsthaft mit der Literatur und dem Denken Goethes auseinandersetzen müsse, mit der Philosophie Kants, Fichtes und Schellings und mit der Theologie Schleiermachers.

Vorangegangen war die Konzentration amerikanischer Universitäten wie Harvard und anderer auf die empirische Philosophie John Lockes, derzufolge die sinnliche Erfahrung Hauptquelle der Erkenntnis sei. Diese Philosophie leistete einen wichtigen Beitrag zum religiösen Liberalismus, indem sie den Gebrauch der Vernunft und Rücksicht auf historische Bedingungen bei der Interpretation von Texten empfahl. Auf dieser Basis lehnten die Unitarier dann die Doktrin der Dreifaltigkeit ebenso ab wie die calvinistische Behauptung einer universalen menschlichen Verderbtheit. Aber der Locke'sche Empirismus hatte auch konservative Eigenschaften. Die erste Generation amerikanischer Unitarier sah die Bibel als den allein maßgeblichen Offenbarungstext an, dessen Autorität durch die Wunder Jesu und der Apostel legitimiert war. Die Wunder wiederum gewannen ihre Autorität eben aus ihrer sinnlichen Erfahrbarkeit.

Ausgehend von Kants Philosophie stellten nun die amerikanischen Transzendentalisten diese Idee auf den Kopf. Für sie ergab sich die maßgebliche Autorität vielmehr aus einer inneren Intuition, nicht aus äußerlichen Wunderzeichen. Weder Wunder noch Schriftwerke konnten die Offenbarung Gottes übertreffen, die sich in der menschlichen Seele vollzieht. In der Folge wandelte sich das liberale Christentum der ersten Generation in einen religiösen Liberalismus, der sich nicht mehr der Geltung einer spezifischen religiösen Tradition zuordnen ließ. Dieser Wandel machte den Weg frei für die Bewegung des religiösen Humanismus, die im 20. Jahrhundert aus ihm hervorging. Wie die Transzendentalisten versucht hatten, religiös zu sein, ohne sich an eine bestimmte historische Gestalt des Christentums zu binden, so glaubten auch die Humanisten an Gott. Heute verstehen sich nach wie vor viele Unitarische Universalisten selber als Christen; sehr viel mehr aber würden sich eher allgemein als Humanisten beschreiben oder als Buddhisten oder 
Heiden oder einfach als spirituell Suchende. Was all diese unterschiedlichen Menschen verbindet, ist die Verpflichtung jedes einzelnen, seine oder ihre jeweils eigenen religiösen Überzeugungen in Freibeit zu erlangen.

Die Transzendentalisten haben die deutschen Ideen nicht passiv absorbiert, sondern sie kreativ an die demokratischen Ideale des modernen Amerika angepasst. Margaret Fuller beispielsweise liebte Eckermanns Gespräche mit Goethe. Aber Goethe wäre möglicherweise überrascht gewesen über ihre Entscheidung, von ihnen ausgehend Gespräche mit jungen Frauen in Boston zu führen, und zwar in aller Öffentlichkeit, was um diese Zeit für Frauen in Neuengland ein Tabu war. Aber Fuller hoffte, dass ein Anflug der Goethe'schen Gesprächskultur den Frauen die nötigen Kenntnisse und den Mut vermitteln könnte, die sie brauchten, um dieses Tabu zu brechen. Viele der Teilnehmerinnen an Fullers Veranstaltungen wurden zu führenden Persönlichkeiten in dem langen Kampf um das Frauenwahlrecht. Auch Theodore Parker war nicht nur ein brillanter Redner und Gelehrter, der die Einsichten der deutschen historischen Bibelkritik aufgriff, sondern zugleich ein beliebter Prediger, der diese Ideen in der Boston Music Hall tausenden von Zuhörern vermittelte. Parkers Gemeinde konnte an einem Sonntag die Grundsätze der historischen Kritik kennenlernen und am nächsten Gedanken zum bewaffneten Widerstand gegen die Sklaverei hören. Parker war einer der nicht wenigen führenden Vertreter des Transzendentalismus, die mithalfen, den amerikanischen Bürgerkrieg als Kampf gegen die Sklaverei zu initiieren, indem sie John Browns Versuch unterstützten, eine große Sklavenrevolte zu entfachen. Andere Transzendentalisten kamen zu der Überzeugung, dass sie Gottes Stimme in der Seele und im Gewissen dann am besten folgen konnten, wenn sie utopische Gemeinden gründeten, in denen alle Menschen zugleich mit den Händen und mit den Köpfen arbeiteten, oder indem sie in den Rhythmus der natürlichen Welt eintauchten. Die transzendentalistische Verbindung deutscher und amerikanischer Ideen und Ideale inspirierte, was man später die Amerikanische Renaissance nannte, eine Blütezeit literarischer und philosophischer Kreativität in der Geschichte Amerikas.

Zeitgenössische Unitarische Universalisten halten diesen Geist des Transzendentalismus in unserer heutigen künstlerischen Kreativität und unseren sozialen und ökologischen Aktivitäten lebendig. Die jüngste Hauptversammlung der Unitarischen Universalisten beispielsweise legte, unter dem programmatischen Namen einer „Gerechtigkeitshauptversammlung“, den Fokus auf die Rechte von Einwanderern. Der Höhepunkt war eine Demonstration von tausenden von Unitarischen Universalisten vor der Justizvollzugsanstalt, in der Migranten oft ohne Zugang zu einer Rechtsvertretung oder zu ihren Familien gefangen gehalten werden. 


\section{Glauben für Gebildete}

Das vierte konstitutive Ereignis der unitarischen Geschichte ist ihre seit jeher enge Verbundenheit mit Bildungsinstitutionen. In vielerlei Hinsicht kann man sagen, dass der amerikanische Unitarismus an der Harvard-Universität geboren wurde. Die Liebe zum Lernen veranlasste die Puritaner nur wenige Jahre nach ihrer Ankunft in Massachusetts, eine Hochschule zu gründen; diese Hochschule war es dann, über die neues Gedankengut aus Europa nach Amerika und in die Ausbildung der Geistlichen gelangte. Im Laufe des gesamten 18. Jahrhunderts gingen immer mehr in Harvard ausgebildete Pfarrer vom Calvinismus zum Gedankengut der Aufklärung über. Als Harvard 1805 einen erklärten Unitarier auf seinen wichtigsten Lehrstuhl berief, reagierten orthodoxe Calvinisten entsetzt - und die daraus resultierende Kontroverse brachte die Unitarier dazu, eine eigene Glaubensgemeinschaft zu organisieren. In den folgenden Jahrzehnten gründeten unitarische Pfarrer in westlichen Städten neue, unserem liberalen Glauben verpflichtete Schulen und Universitäten. Die meisten davon waren nicht ausdrücklich unitarisch, sondern sahen sich einem Geist liberaler Toleranz verpflichtet und waren offen für alle Glaubensrichtungen oder auch für die Möglichkeit, sich gar keiner Glaubensrichtung anzuschließen. In jedem Fall aber hielten sie die unitarische Absicht lebendig, sich intensiv um Bildung zu bemühen.

Diese Verbindung von Unitarismus und Bildungswesen wurde in den Jahren nach dem Zweiten Weltkrieg erneuert. Während dieser Zeit förderte die amerikanische Regierung Veteranen, die an einer Universität studieren wollten; Größe und Anzahl der Hochschulen und Universitäten in Amerika nahmen rasch zu. Zur gleichen Zeit führten die Unitarier das Fellowship Movement ein, das sich zu einer innovativen Strategie für die Gründung neuer Gemeinden entwickelte. Dieses Programm erlaubte es kleinen Gruppen von Laien, lokale Fellowships zu gründen, auch wenn sie nicht groß genug waren, um professionelle Pfarrer einzustellen. Besonders in Universitätsstädten gab es diese Organisationsform. Als ich beispielsweise in Minnesota lebte, gehörte ich einer Fellowship an, die von Professoren der örtlichen Hochschule für Lehramtsstudiengänge gegründet worden war. Viele dieser Professoren waren aus anderen Teilen des Landes hierher gezogen und waren sehr viel liberaler eingestellt als ihre Nachbarn in den ländlichen Gebieten Minnesotas. Die Fellowship vermittelte ihnen ein Gemeinschaftsgefühl und erleichterte zudem eine Verbindung akademischer und religiöser Werte. Obwohl die Fellowships klein anfingen, bilden viele von ihnen jetzt die am schnellsten wachsenden Gemeinschaften innerhalb unserer Bewegung. Weil sie weiterhin wachsen, ist der Unitarische Universalismus als Ganzes in der Lage, während der letzten zwanzig Jahre eine konstante Größe zu wahren, während die meisten anderen liberalen religiösen Traditionen in Amerika rasant an Mitgliedern verlieren. 


\section{Die Vereinigung des Unitarismus mit dem Universalismus}

Ein abschließendes Ereignis in der Geschichte der amerikanischen Unitarier erklärt den etwas merkwürdigen Namen, den wir heute tragen: 1961 vereinigte sich die American Unitarian Association mit der Universalist Church of America, um die Unitarian Universalist Association of Congregations zu bilden. Beide Traditionen teilten seit zwei Jahrhunderten wesentliche liberale Grundsätze. Der Unitarismus war aus der Ablehnung des Trinitätsdogmas hervorgegangen; der Universalismus hatte die Lehre von der Hölle zurückgewiesen; und beide hatten im Laufe ihrer jeweiligen Entwicklung christliche, post- und außerchristliche Strömungen des Liberalismus miteinander zu vereinen. Viele allgemein respektierte Führungspersönlichkeiten waren mit beiden Traditionen eng verbunden. Es dauerte jedoch einige Zeit, ehe eine gemeinsame Organisation entstehen konnte - vor allem weil jede Gruppe ihre eigene, stolze Geschichte erlebt und weil jede dabei sehr unterschiedliche Prägungen erfahren hatte.

Während der Unitarismus von etablierten puritanischen Kirchen abstammt, hatte sich der Universalismus von Beginn an jeder staatlichen Unterstützung von Religion widersetzt. Eine der ersten universalistischen Gemeinden hatte sogar einen Rechtsstreit ausfechten müssen, damit ihre Mitglieder keine Kirchensteuern zu zahlen brauchten; Universalisten waren es auch, die in Massachusetts den erfolgreichen Kampf um eine Änderung der Machtverhältnisse geführt hatten. In derselben Zeit, in der die Unitarier eng mit Hochschulen und Universitäten verbunden waren, tendierten frühe Universalisten zu autodidaktischer Unabhängigkeit. Sie waren entschieden intellektuell, wollten jedoch nicht von Bildungseinrichtungen abhängig sein, die sie in der Gefahr eines unterwürfigen Gehorsams gegenüber traditionellen religiösen Autoritäten sahen. Ein weiterer wichtiger Unterschied hat mit der Weise zu tun, in der Universalisten auf theologische Veränderungen reagiert hatten. Die Unitarier hatten erfolgreich eine in ihren Möglichkeiten breitgefächerte Kirche aufgebaut, die ebenso Raum bot für rebellische neue Ideen wie für den Transzendentalismus deutsch-idealistischer Prägung oder einen atheistischen Humanismus. Der Universalismus produzierte zwar mindestens ebenso viel Rebellentum, seine Rebellen aber wanderten dauerhaft ab. Als Konsequenz erlitt der Universalismus in den sich beruhigenden Zeiten einen stetigen Verlust an Mitgliedern. Er stimmte dem Zusammenschluss mit den Unitariern im Wesentlichen $\mathrm{zu}$, weil es keineswegs gesichert schien, dass er allein überlebensfähig bleiben könnte.

Während der letzten fünfzig Jahre hat das Erbe der Universalisten im erheblichen Maße zur Lebendigkeit des Unitarischen Universalismus beigetragen. Einige der besten Arbeiten, die sich in letzter Zeit mit der Geschichte der Unitarischen Universalisten beschäftigt haben, konzentrieren sich auf den Universalismus, weil diese Tradition in der Vergangenheit vernachlässigt wurde. Der Universalismus hält ein Gegengift gegen einige der problematischen Aspekte des Unitarismus bereit, insbesondere im Hinblick auf dessen ökonomisches und bildungsorientiertes 
Elite-Denken. Universalisten betonen, dass die unendliche Liebe Gottes den geistig Suchenden anspricht, und sie fühlen sich abgestoßen von dem, was sie als einen trockenen Rationalismus der Unitarier empfinden. Viele junge Leute, auch viele meiner eigenen Studenten, sehen sich selbst als Universalisten, auch wenn sie in Gemeinden aufgewachsen sind, die aus der unitarischen Seite der Vereinigung hervorgegangen sind. In den letzten Jahren wurden universalistische Glaubenslehren auch von evangelischen Christen wiederentdeckt, die zu der Überzeugung gelangten, dass beispielsweise die Doktrin einer ewigen Verdammnis nicht in der Bibel zu finden sei. Unitarische Universalisten arbeiten konsequent daran, einen Raum für bibeltreue Mitglieder innerhalb ihrer Gemeinschaften zu schaffen, Seite an Seite mit Buddhisten, Heiden, Atheisten.

Geschichtliche Ereignisse wie die hier skizzierten können jeder religiösen Tradition Vorteile bringen. Die meisten religiösen Liberalen würden zu Recht immer noch darauf bestehen, dass die Vergangenheit keine Macht über die Gegenwart haben sollte. Aber eine Vergangenheit, die keine Macht darstellt, kann trotzdem ein Freund sein. Die amerikanischen Unitarier jedenfalls, zu denen auch ich mich zähle, haben unsere puritanischen Vorfahren, unsere transzendentalen Rebellen und unsere universalistischen Schwestern und Brüder dazu gebracht, sehr wertvolle und hilfreiche Freunde für uns zu sein. 